\title{
Geometric phase and band inversion in periodic acoustic systems
}

\author{
Meng Xiao ${ }^{1,2 \dagger}$, Guancong Ma ${ }^{1,2 \dagger}$, Zhiyu Yang ${ }^{1}$, Ping Sheng ${ }^{1,2}$, Z. Q. Zhang ${ }^{1,2}$ and C. T. Chan ${ }^{1,2 \star}$
}

\begin{abstract}
The geometric-phase concept has far-reaching implications in many branches of physics ${ }^{1-14}$. The geometric phase that specifically characterizes the topological property of bulk bands in one-dimensional periodic systems is known as the Zak phase $^{15,16}$. Recently, it has been found that topological notions can also characterize the topological phase of mechanical isostatic lattices ${ }^{13}$. Here, we present a theoretical framework and two experimental methods to determine the Zak phase in a periodic acoustic system. We constructed a phononic crystal with a topological transition point in the acoustic band structure where the band inverts and the Zak phase in the bulk band changes following a shift in system parameters. As a consequence, the topological characteristics of the bandgap change and interface states form at the boundary separating two phononic crystals having different bandgap topological characteristics. Such acoustic interface states with large sound intensity enhancement are observed at the phononic crystal interfaces.
\end{abstract}

We use a simple photonic crystal (PC) system to demonstrate geometric phase (GP) effects and the existence of topological transition points in acoustic systems. The experimental set-up is shown in Fig. 1a. The PC is a cylindrical waveguide with periodically alternating cross-sectional areas. Each unit cell has two wider tubes (tube-A) of length $(1 / 2) d_{\mathrm{A}}$ and inner radius $r_{\mathrm{A}}=2.4 \mathrm{~cm}$, sandwiching a narrower tube-B of length $d_{\mathrm{B}}$ and inner radius $r_{\mathrm{B}}=1.5 \mathrm{~cm}$. The tubes are filled with air (mass density $\rho=1.3 \mathrm{~kg} \mathrm{~m}^{-3}$, speed of sound $v=343 \mathrm{~m} \mathrm{~s}^{-1}$ ) and made of hard plastics to ensure that the inner surfaces meet the sound hardboundary condition. An example of a unit cell, with $d_{\mathrm{A}}=3.0 \mathrm{~cm}$ and $d_{\mathrm{B}}=5.5 \mathrm{~cm}$ (we refer to this configuration as ' $\mathrm{S} 1$ '), is shown in Fig. $1 b-d$, together with simulated pressure eigenfunctions of the lowest three eigenmodes at $k=0$. Their corresponding eigenfrequencies are marked by red dots in Fig. 1e, which shows the acoustic band structure ${ }^{17-19}$. It is clear that the lower two modes have their pressure gradient along the propagation direction $k$ (Fig. 1b,c) and negligible pressure variation along the cross-sectional directions. These two modes represent the two band-edge states of the second bandgap between the second and third bands, as shown by black curves in Fig. 1e. The mode shown in Fig. 1d has a clear pressure variation in the cross-sectional planes. The dispersion of this mode is marked by the green line in Fig. 1e. For symmetry reasons, this mode cannot be excited with a plane wave, and is therefore not considered in the following discussions.

The two longitudinal modes can be further labelled as even (Fig. 1b) and odd (Fig. 1c) with respect to the centre of tube$\mathrm{A}$ at $k=0$. The eigenfrequencies of these two band-edge states can be tuned by varying $\Delta d \equiv\left(d_{\mathrm{A}}-d_{\mathrm{B}}\right) / 2$ (while keeping $r_{\mathrm{A}}, r_{\mathrm{B}}$ and the total length $a=d_{\mathrm{A}}+d_{\mathrm{B}}$ constant). Their eigenfrequencies as a function of $\Delta d$ are plotted in Fig. 2. The results show that it is possible to tune the eigenfrequencies of the two band-edge states to cross each other, with accidental degeneracy occurring at $\Delta d=0.49 \mathrm{~cm}$. The gap closing and reopening and the corresponding switching of the two band-edge states are analogous to the band inversion process in electronic systems $\mathrm{s}^{20-22}$, with $\Delta d=0.49 \mathrm{~cm}$ corresponding to a topological transition point in our acoustic system.

The parallel can be further seen by calculating the Zak phases of our PC systems. Originally defined for electronic systems, the Zak phase is a special type of Berry phase in one-dimensional (1D) periodic systems ${ }^{15,23}$. We extend the concept of the Zak phase to acoustics as follows. The GP for the $n$th isolated band is given by

$$
\theta_{n}^{Z a k}=\int_{-\pi / a}^{\pi / a}\left[i \int_{\text {unitcell }} \frac{1}{2 \rho v^{2}} \mathrm{~d} \mathbf{r} \mathrm{d} x u_{n, k}^{*}(x, \mathbf{r}) \partial_{k} u_{n, k}(x, \mathbf{r})\right] \mathrm{d} k
$$

where $x$ is the axial coordinate, $\mathbf{r}$ represents positions in a crosssectional plane, $\rho$ is the density of air, $v$ is the speed of sound in air and $u_{n, k}(x, \mathbf{r})$ is the periodic in-cell part of the normalized Bloch pressure eigenfunction of a state in the $n$th band with wavevector $k$-that is, $P_{n, k}(x, \mathbf{r})=u_{n, k}(x, \mathbf{r}) \exp (i k x)$. The factor $1 /\left(2 \rho v^{2}\right)$ is the weight function of an acoustic system and is a constant in our system. On the other hand, with the pressure field being uniform in the lateral dimension, our system can be regarded as a quasi$1 \mathrm{D}$ system ${ }^{17-19}$. The Zak phase of an isolated band in such systems can also be obtained from the symmetry properties of band-edge states $^{15,23}$. As the unit cell has mirror symmetry with respect to its central cross-sectional plane, the Zak phase can have only two values: 0 or $\pi$ (see Supplementary Information I). If two states at the centre and edge of the Brillouin zone belonging to the same band have the same symmetry - that is, both even or odd with respect to the central plane of tube-A-then the Zak phase of this band is 0 . Otherwise, the Zak phase is $\pi$.

We next investigate four different PC configurations, with each having the same unit cell length $a=8.5 \mathrm{~cm}$ but a different $\Delta d$. These PC configurations are denoted as S1, S2, S3 and S4, and their corresponding $\Delta d$ values are indicated by vertical dashed lines in Fig. 2. Specifically, $\Delta d=-1.25 \mathrm{~cm}$ for $\mathrm{S} 1,2.25 \mathrm{~cm}$ for S2, $-0.25 \mathrm{~cm}$ for S3, and $0.49 \mathrm{~cm}$ for S4. Figure 3 shows the calculated band structures of these four PCs. The Zak phases of each PC are calculated numerically using equation (1) and are labelled in red in Fig. 3. $\theta_{2}^{\text {Zak }}=\pi$ for the second band of both $S 1$ and $S 3$, whereas $\theta_{2}^{Z \mathrm{Zak}}=0$ for the second band of S2. The second bandgap of S4

\footnotetext{
${ }^{1}$ Department of Physics, Hong Kong University of Science and Technology, Clear Water Bay, Kowloon, Hong Kong. ${ }^{2}$ Institute for Advanced Study, Hong Kong University of Science and Technology, Clear Water Bay, Kowloon, Hong Kong. 'These authors contributed equally to this work.

*e-mail: phchan@ust.hk
} 

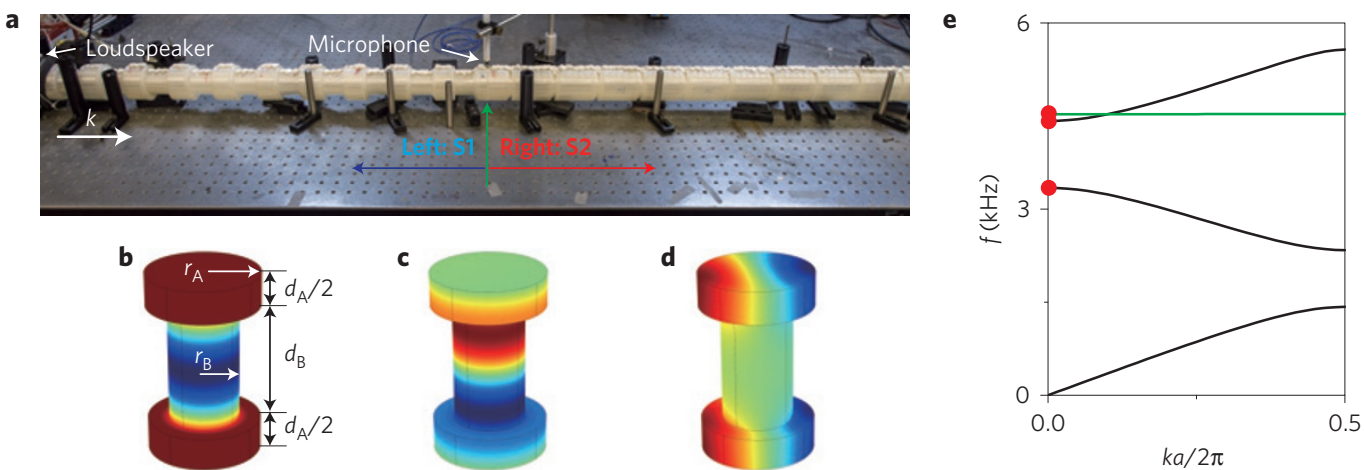

Figure 1 | 1D phononic crystal interface system. a, Experimental set-up. The green arrow marks the position of the interface separating the PC with configuration S1 on the left and the PC with configuration S2 on the right. The large white arrow shows the incidence direction. $\mathbf{b}$-d, Simulated pressure eigenfunctions of an $\mathrm{S} 1 \mathrm{unit}$ cell. b, Lowest even mode, located at 3,341 Hz. c, Odd mode, located at 4,421 Hz. d, First transverse mode, located at 4,527 Hz. Red/blue colour indicates positive/negative local pressure. Eigenfunctions of the PC configurations S2, S3 and S4 are qualitatively similar, but have different eigenfrequencies. e, Band structure of S1, where the black lines represent fundamental modes and the green line a higher order mode. Red dots mark the eigenfrequencies shown in b-d. The higher order mode marked by the green line is a 'deaf mode' that cannot be excited by the incident plane wave.

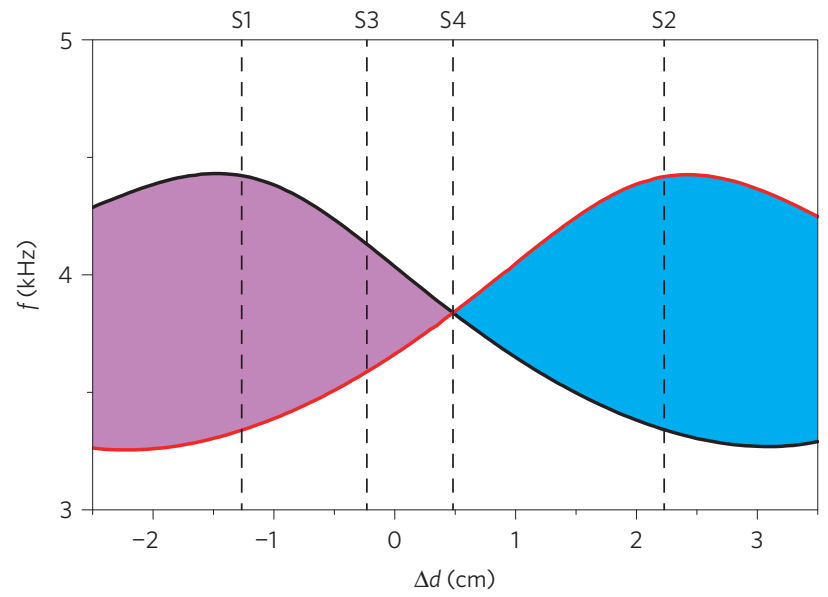

Figure 2 | Topological transition in 1D PC system. The eigenfrequency of eigenstates bounding the second bandgap (coloured regions) at the centre of the Brillouin zone as a function of $\Delta d=\left(d_{A}-d_{B}\right) / 2$. The red and black curves correspond to even and odd modes (with respect to the central cross-sectional plane of tube- $A$ ). As the length difference $\Delta d$ increases, the second bandgap closes and reopens, accompanied by the crossing of two eigenmodes. This process is analogous to the band inversion process; thus the second bandgaps are marked with different colours to show that they have different topological characteristics. Four different configurations are considered, with the length difference $\Delta d$ indicated with vertical dashed lines. It can be seen that the second bandgaps of configurations S1 and S3 are topologically identical, and differ from that of S2. The second bandgap of S4 closes at the centre of the Brillouin zone.

closes at the zone centre where the two modes become degenerate at $k=0$ (Fig. $3 \mathrm{c}$ ). This marks a topological transition point. This is also shown in Fig. 2, where a gap closing and reopening process can be seen if the parameters of the system are tuned continuously from those of S1 or S3 to those of S2, passing through a transition point at $\Delta d=0.49 \mathrm{~cm}$. Furthermore, the system's mirror symmetry is preserved when we vary $\Delta d$, and none of the first bandgaps of the four PCs closes during this process. It follows that the first bandgaps of these four systems remain topologically identical. Note that the topological property of a bandgap is determined by the summation of the Zak phases of all the bands below this gap, but has no dependence on the properties of the higher bands ${ }^{24,25}$. For S1 and S $3, \theta_{2}^{\mathrm{Zak}}=\pi$, and so the topological characters of their first and second bandgaps are identical. In contrast, $\theta_{2}^{\text {Zak }}=0$ for S2, indicating that the second bandgap of S2 is topologically different from that of S1 or S3.

The determination of the GP has been theoretically proposed and experimentally done in cold atom ${ }^{16,26}$ and photonic systems $s^{27,28}$. In our acoustic system, we follow a different scheme to determine the GP experimentally. The bulk band GP can be determined by measuring the reflection phase $\phi$ at the boundary of the PC. In a quasi-1D system, $\phi$ of the reflected pressure field of a PC for frequencies inside the bandgap is a manifestation of its Zak phase $^{24,25}$. The reflection phase satisfies $\phi \in\{-\pi, 0\}$ or $\phi \in\{0, \pi\}$ $(\bmod 2 \pi)$ inside a bandgap, depending on the topological character of that gap ${ }^{25}$. The topological character of a gap is related to the symmetry types (even or odd) of the band-edge states below or above this gap, while the Zak phase yields the relationship between the two band-edge states of a band. Thus, the Zak phase of a bulk band and the topological characters of the two bandgaps sandwiching this band can be related to each other through the symmetry types of the band-edge states. Owing to the inversion symmetry that is inherent in the system under consideration, the geometric Zak phase can take only two values: either 0 or $\pi$ (see Supplementary Information I). It has been shown rigorously that the relationship between $\operatorname{sgn}(\phi)$ of the first and second bandgaps and the Zak phase of the second band is given by ${ }^{24}$.

$$
\operatorname{sgn}\left(\phi_{1}\right) / \operatorname{sgn}\left(\phi_{2}\right)=-\exp \left(i \theta_{2}^{\text {Zak }}\right) \text {, with } \theta_{2}^{\text {Zak }}=0 \text { or } \pi
$$

where the subscripts indicate the number of bandgaps. In other words, measuring the signs of the reflection phases of the two bandgaps sandwiching an isolated bulk band provide sufficient information to determine the Zak phase of that band. If the signs are the same, the Zak phase of that band is $\pi$. Otherwise, it is zero. This is the first method we use to measure the Zak phase of the bulk bands. For the measurement, we add a homogeneous waveguide with an identical inner radius to that of tube-A between the loudspeaker and the PC. Figure 4a shows a schematic drawing of the set-up used to measure the reflection phase. The measured fields in the second common bandgaps of S1, S2 and S3 are shown in Fig. 4b, together with that of a flat sound hard surface (steel plate) for reference. The reflection fields of S1 and S3 seem to be 'advanced' with respect to the steel plate, whereas the reflection field of S2 seems to be 'delayed'. Thus $\phi_{2}$ values for the two topologically identical PCs-that is, S1 and S3-have the same sign, whereas $\phi_{2}$ for S2 has the opposite sign. In comparison, the measured reflection phases of the first common bandgaps, $\phi_{1}$, of all three PCs have the same sign (Fig. 4c). Excellent agreement with the theoretical prediction is seen. 
a

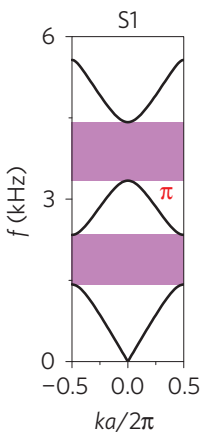

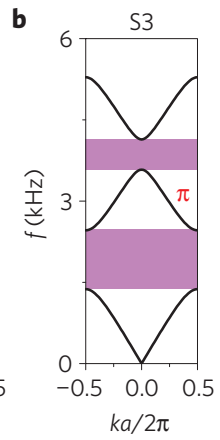
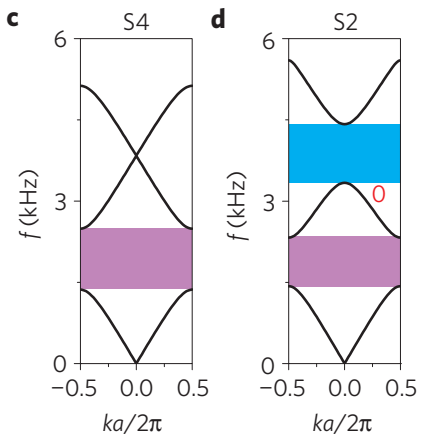

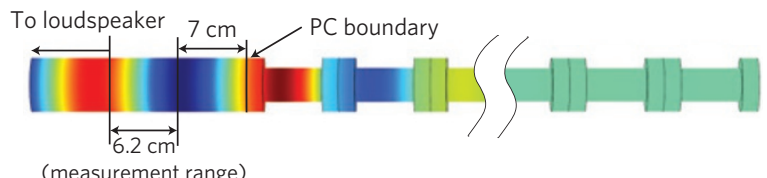

Figure 3 | Topological transition with Zak phase changes. a-d, The band structures (black lines) of configurations S1, S3, S4 and S2, respectively. The regions in magenta and the region in cyan mark bandgaps with different topological characteristics. The first bandgaps of S1-S4 (all in magenta) are topologically identical, as are the second bandgaps of $\mathrm{S} 1$ and S3 (also in magenta). The second bandgap of S2 is topologically different from those of S1 and S3. The topological properties of bandgaps are related to the Zak phases of bulk bands below these gaps. The Zak phase of the second band is labelled in red. The second bands of S1 and S3 have Zak phase $\pi$ whereas the second band of $\mathrm{S} 2$ has Zak phase zero, which indicates the existence of a transition point. S4 is at the transition point, where the second bandgap closes at the centre of the Brillouin zone.

To further corroborate our conclusion, we use a second method to determine the Zak phase: by directly measuring the symmetry of the band-edge states ${ }^{23,25}$. Direct measurement of bulk states is relatively straightforward in an acoustic wave system. We made point-by-point measurements of the pressure distribution inside a unit cell in the bulk PC; the experimental results are shown in detail in the Supplementary Information II. Simply put, we found that for $\mathrm{S} 1$ and S3, the acoustic modes at $k=0$ and $k=\pi / a$ have different symmetries in the second band, meaning that the Zak phase must be $\pi$ (ref. 24). For S2, the acoustic modes at $k=0$ and $k=\pi / a$ have the same symmetry in the second band, meaning that the Zak phase must be 0 (ref. 24). These results agree with the prediction of equation (2) using the reflection phase measurements. To the best of our knowledge, this is the first experimental determination of GP in the band structure of an acoustic system.

Although Zak phases are geometric properties of the bulk bands, they have important consequences at interfaces. With the bulk Zak phases of the second band determined, we can predict the presence or absence of interface modes at the boundary of different PC configurations ${ }^{24}$. In our system, there must be an interface mode with a frequency inside the second common bandgap at the boundaries separating S1 and S2, and S3 and S2, which differ in their topological properties. In contrast, no such mode can exist in S1 and S3. A simulated distribution of the pressure at the PC interface between S1 and S2 is shown in Fig. 5a, where the green arrow indicates an interface mode predicted from bulk band GP considerations. In Fig. 5b-d, we show respectively the experimentally measured pressure response spectra at the interfaces of configurations $\mathrm{S} 1+\mathrm{S} 2, \mathrm{~S} 3+\mathrm{S} 2$ and $\mathrm{S} 1+\mathrm{S} 3$, together with the corresponding numerical simulations. Sharp transmission peaks are clearly observed for $\mathrm{S} 1+\mathrm{S} 2$ and $\mathrm{S} 3+\mathrm{S} 2$ at $3,836 \mathrm{~Hz}$ and $3,814 \mathrm{~Hz}$ respectively (Fig. 5b,c). These frequencies fall inside their second common bandgap. Such a peak is absent for $\mathrm{S} 1+\mathrm{S} 3$ (Fig. $5 \mathrm{~d}$ ). These observations are consistent with predictions. For $\mathrm{S} 1+\mathrm{S} 2$, we further measured the spatial distribution of the pressure field at the transmission peak frequency of $3,836 \mathrm{~Hz}$. The result is shown in Fig. 5e. It can be seen that the pressure amplitude decays rapidly away from the PC interface (chosen to be $x=0$ ) - a clear sign that the transmission peak appeared as a result of the interface state (details can be found
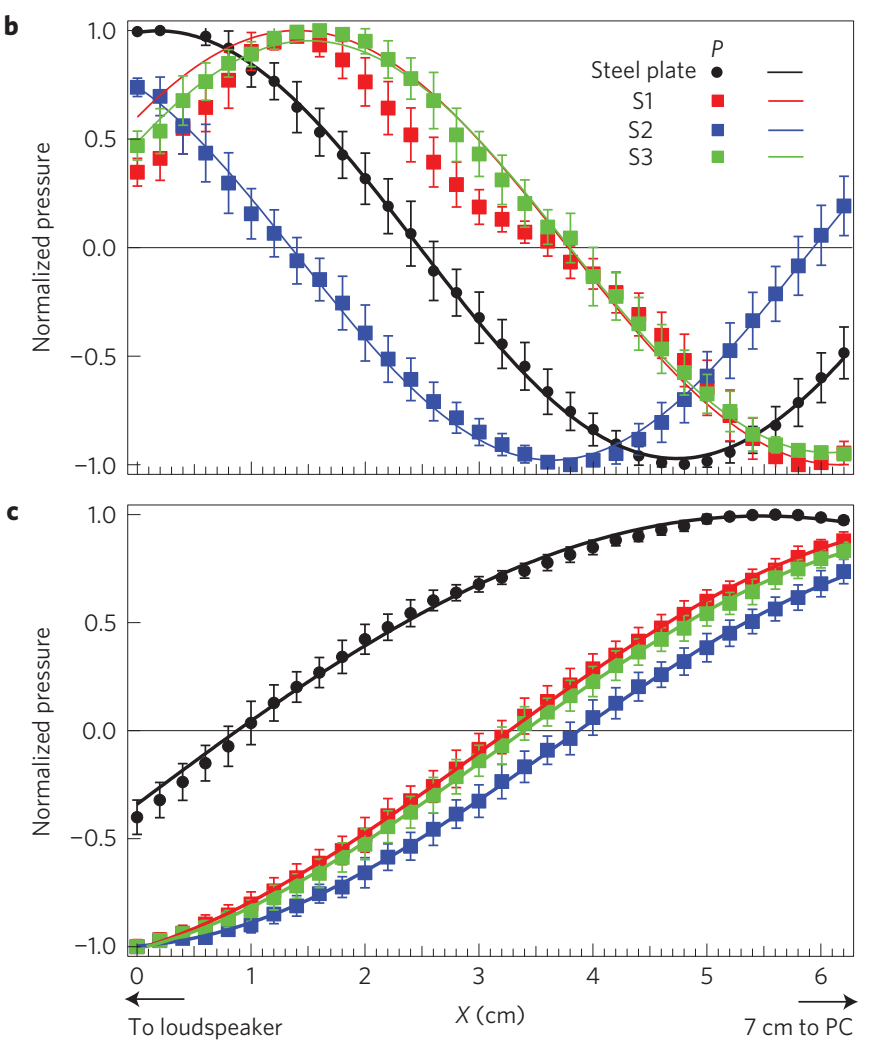

Figure 4 | Reflection phase as a measure of the Zak phase. a, Schematic drawing of the system set-up for measuring the reflection phase, where the $6 \mathrm{~cm}$ range of a measurement tube is shown. $\mathbf{b}$, Self-normalized reflected pressure field measured at $3,836 \mathrm{~Hz}$-that is, inside the second bandgaps of the S1, S2 and S3 PCs. This is also the interface state eigenfrequency of configuration S1+S2. c, The same quantity measured at 2,100 Hz, which is inside the first bandgaps of all three PCs. Red and blue squares are for $\mathrm{S1}$ and $\mathbf{S 2}$, respectively, whereas green triangles are for $\mathbf{S 3}$. As a reference, the reflected pressure field of a steel plate is also shown as black circles. Solid curves in $\mathbf{b}$ and $\mathbf{c}$ of corresponding colours are numerically fitted (with a sine function) to the measured data. In $\mathbf{b}$, the reflected phases for S1 and S3 have the same sign, which is opposite to that for S2. Phases of all three PCs in the first bandgap, however, have the same sign as shown in $\mathbf{c}$. This is clear evidence that the second bandgaps of S1 and S3 share common topological properties, whereas the bandgap of S2 has a different set of topological properties. Error bars in $\mathbf{b}, \mathbf{c}$ are determined based on the uncertainty in measurement position due to finite size of the microphone.

in Supplementary Information III). Recently, some zero-frequency mechanical floppy modes similar to the interface states found here have been reported in certain isostatic lattices-that is, lattices comprising mass points connected by central-force springs on the verge of mechanical instability. These floppy modes are localized at the domain wall between two isostatic lattices with distinct topological properties ${ }^{13}$.

Knowledge of the bulk band GP in a PC allows us to create interface states which in turn have useful implications. For example, we can think of it as a recipe for acoustic field enhancement. Owing to the highly confined spatial characteristics, the sound intensity can build up at the interface states. As an example, we show the amplification of sound intensity using the interface of 
a

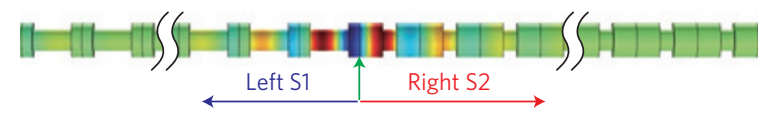

b

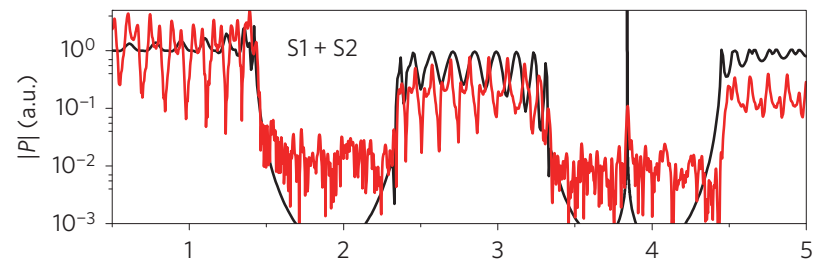

c

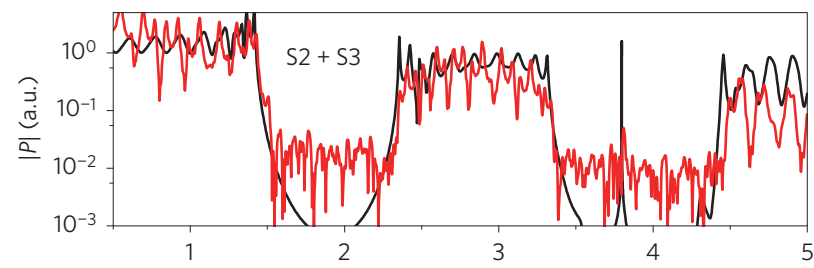

d

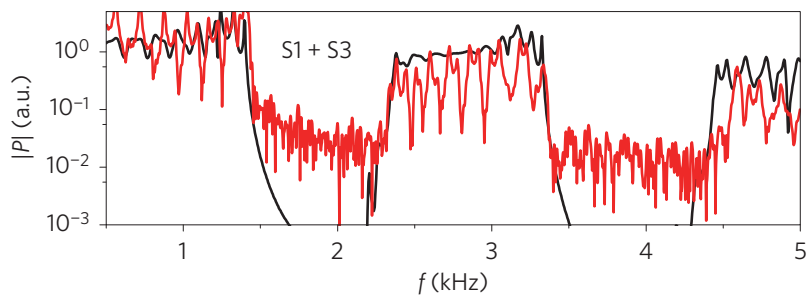

e

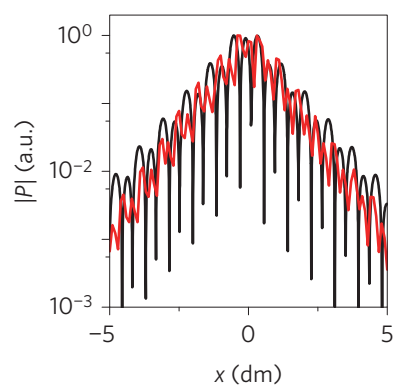

f

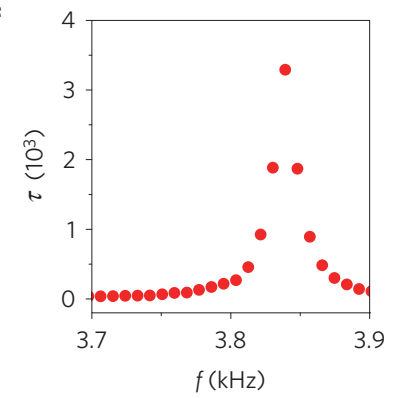

Figure 5 | Existence of interface states in topologically different PC systems. a, Simulated pressure field of the interface state. $\mathbf{b}-\mathbf{d}$, Pressure response spectra at $P C$ interfaces. The system configurations are S1 (left) + S2 (right) (b), S2 + S3 (c) and S1 + S3 (d). All PCs have ten unit cells. Red and black curves indicate experimental and numerical results, respectively. In $\mathbf{b}, \mathbf{c}$, sharp transmission peaks near $3.8 \mathrm{kHz}$ inside the common gap indicate interface states, which are a consequence of the topological difference between S1/S3 and S2. In contrast, no such state is observed in $\mathbf{d}$. This is clear evidence that S1 and S3 are topologically identical. e, Spatial distribution of the amplitude of the pressure field of the $\mathrm{S} 1+\mathrm{S} 2$ system (ten units each) at $3,836 \mathrm{~Hz}$, with the interface located at $x=0$. Red and black curves indicate experimental and numerical results, respectively. Good agreement between experiments and simulations is seen. $\mathbf{f}$, Ratio $(\tau)$ of the measured sound intensity at the interface state to the measured sound intensity in the free-space sound field. An amplification by a factor of over 3,400 is observed.

the $\mathrm{S} 1+\mathrm{S} 2$ system in Fig. 5f. The enhancement ratio $\tau=\left|P_{\mathrm{c}}\right|^{2} /\left|P_{\mathrm{f}}\right|^{2}$ exceeds 3,400 , where $P_{c}$ is the pressure at the PC interface and $P_{f}$ is the pressure in free space. Note that, in this measurement, the PC system is excited directly at the interface (see Supplementary Information III). The enhancement ratio can be further increased if the loss and leakage of sound are reduced in our system. Acoustic field enhancement has obvious applications where strong sound intensities are required, such as sound detection ${ }^{29}$ and biomedical imaging $^{30}$ : a PC system with such an interface state can be placed in close proximity to a sensor/receiver. The local sound intensity can be amplified at the interface, leading to increased sensitivity of the device at the targeted frequency.

We have shown that a periodic acoustic system is an excellent platform to realize advanced concepts such as band inversion and topological transition. The experimental samples can be made with minimal cost and the results are intuitively easy to understand. The acoustic system is rather unique in the sense that reflection phases at boundaries can be measured straightforwardly and, at the same time, the 'field' quantity (which is the sound intensity) can be measured point-by-point inside the sample. These attributes made possible the straightforward determination of the geometric phase of a band. The present results pertain to systems that are periodic in one direction. As it is technically feasible to use $3 \mathrm{D}$ printing to fabricate periodic acoustic crystals that are periodic in two or three dimensions, the periodic acoustic system could be readily employed to explore and determine the relationship between the symmetry of the eigenmodes, geometric phases of the bands and the bulkinterface correspondence in higher dimensions ${ }^{31}$.

\section{Methods}

Experiments. All PC samples were fabricated using 3D printing. Polylactic acid, a kind of hard plastic, was the material chosen to construct the unit cells. The tube walls were printed with sufficient thickness to ensure a suitable rigidity to reproduce a sound hard boundary. A series of small side ports were opened to house the microphone for the measurement of the spatial pressure distribution (Fig. 5e). The ports were sealed with air-tight putty when not in use. The PCs were 3D-printed one unit cell at a time, and were connected with super glue, with the joints further sealed with vacuum grease to minimize loss. S1, S2 and S3 each consisted of ten unit cells. For measurements of the pressure response spectra, a pulse with a central frequency of $3,840 \mathrm{~Hz}$ was generated with an arbitrary waveform generator (Agilent 33220A). The pulse was fed into a loudspeaker as the sound source. In this case the system was excited from its end. The pressure signals were picked up at the PC interface, using a 1/4-inch microphone (PCB Piezotronics Model-378C10), and then recorded with a digital oscilloscope (Agilent DSO6012A). The measurements were typically repeated up to 256 times with a $0.5 \mathrm{~s}$ repetition period, and then averaged to minimize random errors and obtain a highly stable readout. A Fourier transform was then performed to obtain the pressure response as a function of frequency. The relatively shallower depths of bandgaps in Fig. 5b-d are attributed to the noise level of our set-up. However, this does not affect our arguments and conclusion.

For the measurement of the spatial pressure distribution and sound intensity amplification, a small port was opened at the interface and connected to the loudspeaker. The loudspeaker was driven by a lock-in amplifier (Stanford Research SR850) to generate a monochromatic signal with the same frequency as the interface state $(3,836 \mathrm{~Hz})$. The pressure field was manually mapped by moving the microphone across each of the side ports on the PCs. The measured signal was also detected by the same lock-in amplifier. With a sufficient time constant, the readout is highly stable. The sound intensity amplification was determined by removing the PC interface while keeping the sound source and the microphone in place. For the measurement of the reflection phase, each system was also excited with a monochromatic sound $(3,836 \mathrm{~Hz}$ for the second bandgap, and $2,100 \mathrm{~Hz}$ for the first bandgap). The pressure field, together with the relative phase (with respect to a reference signal), was then manually mapped. In measurements of the spatial pressure distribution, the finite size of the microphone (the diaphragm diameter is $\sim 4 \mathrm{~mm}$ ) introduces an uncertainty in position $\leq 2 \mathrm{~mm}$, which then propagates into the measured pressure. Error bars in experimental data were calculated based on this uncertainty.

Simulations. All simulations were performed using the 'Acoustics module' of COMSOL Multiphysics, a commercial finite-element solver package. A full three-dimensional geometry was used. Air (density $\rho=1.3 \mathrm{~kg} \mathrm{~m}^{-3}$, speed of sound $v=343 \mathrm{~m} \mathrm{~s}^{-1}$ ) is chosen as the fluid medium. Eigenmode calculations were carried out to find the pressure eigenfunctions of the PC unit cells as well as their band structures. Frequency domain calculations were performed to obtain the pressure response spectra. To calculate the Zak phase, we used a discretized form of equation (1) as follows:

$$
\theta_{n}^{\mathrm{Zak}}=-\operatorname{Im} \sum_{i=1}^{N} \ln \left[\frac{1}{2 \rho v^{2}} \int_{\text {unit cell }} \mathrm{d} \mathbf{r} \mathrm{d} x u_{n, k_{i}}^{*}(x, \mathbf{r}) u_{n, k_{i+1}}(x, \mathbf{r})\right]
$$

where we have selected $N$ points from $k=\pi / a$ to $k=-\pi / a$. Equation (3) gives the Zak phase in the limit $\Delta k_{i}=k_{i+1}-k_{i} \rightarrow 0$. The periodic gauge was applied in the calculation of the Zak phase, implying that $P_{n, k=\pi / a}(x, \mathbf{r})=P_{n, k=-\pi / a}(x, \mathbf{r})$ for the two eigenmodes at the edges of the Brillouin zone. For each isolated band, we first calculated the pressure eigenfunction using the eigenmode analysis module 
of COMSOL, and then normalized the eigenfunction with the orthogonal relationship $\int_{\text {unit cell }} \mathrm{d} \mathbf{r} \mathrm{d} x\left(1 / 2 \rho v^{2}\right)\left|P_{n, k}(x, \mathbf{r})\right|^{2}=1$ to obtain the periodic-in-cell part of the eigenpressure $u_{n, k}(x, \mathbf{r})$.

\section{Received 5 August 2014; accepted 11 December 2014;} published online 23 February 2015

\section{References}

1. Von Bergmann, J. \& von Bergmann, H. Foucault pendulum through basic geometry. Am. J. Phys. 75, 888-892 (2007).

2. Xiao, D., Chang, M-C. \& Niu, Q. Berry phase effects on electronic properties. Rev. Mod. Phys. 82, 1959-2007 (2010).

3. Karplus, R. \& Luttinger, J. Hall effect in ferromagnetics. Phys. Rev. 95, 1154-1160 (1954).

4. Kohn, W. \& Luttinger, J. Quantum theory of electrical transport phenomena. Phys. Rev. 108, 590-611 (1957).

5. Chang, M-C. \& Niu, Q. Berry phase, hyperorbits, and the Hofstadter spectrum. Phys. Rev. Lett. 75, 1348-1351 (1995).

6. King-Smith, R. \& Vanderbilt, D. Theory of polarization of crystalline solids. Phys. Rev. B 47, 1651-1654 (1993).

7. Thonhauser, T., Ceresoli, D., Vanderbilt, D. \& Resta, R. Orbital magnetization in periodic insulators. Phys. Rev. Lett. 95, 137205 (2005).

8. Thouless, D., Kohmoto, M., Nightingale, M. \& Den Nijs, M. Quantized Hall conductance in a two-dimensional periodic potential. Phys. Rev. Lett. 49, 405-408 (1982).

9. Wang, Z., Chong, Y., Joannopoulos, J. \& Soljačić, M. Observation of unidirectional backscattering-immune topological electromagnetic states. Nature 461, 772-775 (2009).

10. Rechtsman, M. C. et al. Photonic Floquet topological insulators. Nature 496, 196-200 (2013)

11. Rechtsman, M. C. et al. Topological creation and destruction of edge states in photonic graphene. Phys. Rev. Lett. 111, 103901 (2013).

12. Khanikaev, A. B. et al. Photonic topological insulators. Nature Mater. 12, 233-239 (2012).

13. Kane, C. L. \& Lubensky, T. C. Topological boundary modes in isostatic lattices. Nature Phys. 10, 39-45 (2014).

14. Heeger, A. J., Kivelson, S., Schrieffer, J. R. \& Su, W. P. Solitons in conducting polymers. Rev. Mod. Phys. 60, 781-850 (1988).

15. Zak, J. Berry's phase for energy bands in solids. Phys. Rev. Lett. 62 , 2747-2750 (1989).

16. Atala, M. et al. Direct measurement of the Zak phase in topological Bloch bands. Nature Phys. 9, 795-800 (2013).

17. Bradley, C. Time harmonic acoustic Bloch wave propagation in periodic waveguides. Part I. Theory. J. Acoust. Soc. Am. 96, 1844-1853 (1994).

18. Hu, X., Hang, Z., Li, J., Zi, J. \& Chan, C. Anomalous Doppler effects in phononic band gaps. Phys. Rev. E 73, 015602 (2006).

19. Munday, J., Bennett, C. B. \& Robertson, W. Band gaps and defect modes in periodically structured waveguides. J. Acoust. Soc. Am. 112, 1353-1358 (2002).
20. Hasan, M. Z. \& Kane, C. L. Colloquium: Topological insulators. Rev. Mod. Phys. 82, 3045-3067 (2010)

21. Bernevig, B. A., Hughes, T. L. \& Zhang, S-C. Quantum spin Hall effect and topological phase transition in HgTe quantum wells. Science 314, 1757-1761 (2006)

22. Pankratov, O., Pakhomov, S. \& Volkov, B. Supersymmetry in heterojunctions: Band-inverting contact on the basis of $\mathrm{Pb}_{1-x} \mathrm{Sn}_{x} \mathrm{Te}_{\mathrm{and}} \mathrm{Hg}_{1-x} \mathrm{Cd}_{x} \mathrm{Te}$. Solid State Commun. 61, 93-96 (1987).

23. Kohn, W. Analytic properties of Bloch waves and Wannier functions. Phys. Rev. 115, 809-821 (1959)

24. Xiao, M., Zhang, Z-Q. \& Chan, C. T. Surface impedance and bulk band geometric phases in one-dimensional systems. Phys. Rev. X 4, 021017 (2014).

25. Zak, J. Symmetry criterion for surface states in solids. Phys. Rev. B 32, 2218-2226 (1985)

26. Jotzu, G. et al. Experimental realization of the topological Haldane model with ultracold fermions. Nature 515, 237-240 (2014).

27. Zeuner, J. M. et al. Probing topological invariants in the bulk of a non-Hermitian optical system. Preprint at http://arxiv.org/abs/1408.2191 (2014).

28. Longhi, S. Zak phase of photons in optical waveguide lattices. Opt. Lett. 38, 3716-3719 (2013)

29. Buckingham, M. J., Berknout, B. V. \& Glegg, S. A. Imaging the ocean with ambient noise. Nature 356, 327-329 (1992).

30. Fatemi, M. \& Greenleaf, J. F. Ultrasound-stimulated vibro-acoustic spectrography. Science 280, 82-85 (1998).

31. Huang, X., Xiao, M., Zhang, Z-Q. \& Chan, C. Sufficient condition for the existence of interface states in some two-dimensional photonic crystals. Phys. Rev. B 90, 075423 (2014)

\section{Acknowledgements}

G.M. thanks Ke Sun for his technical support with measurements and signal processing. M.X. thanks Mengyuan He for his help with the numerical calculation of the Zak phase. This work was supported by the Hong Kong Research Grants Council (Grant No. AoE/P-02/12).

\section{Author contributions}

M.X., Z.Q.Z. and C.T.C. provided the theoretical framework. M.X. carried out the numerical simulations. G.M. fabricated the samples and carried out experimental measurements. M.X., G.M., Z.Q.Z. and C.T.C. wrote the manuscript. All authors were involved in the analysis and discussion of the results.

\section{Additional information}

Supplementary information is available in the online version of the paper. Reprints and permissions information is available online at www.nature.com/reprints.

Correspondence and requests for materials should be addressed to C.T.C.

\section{Competing financial interests}

The authors declare no competing financial interests. 